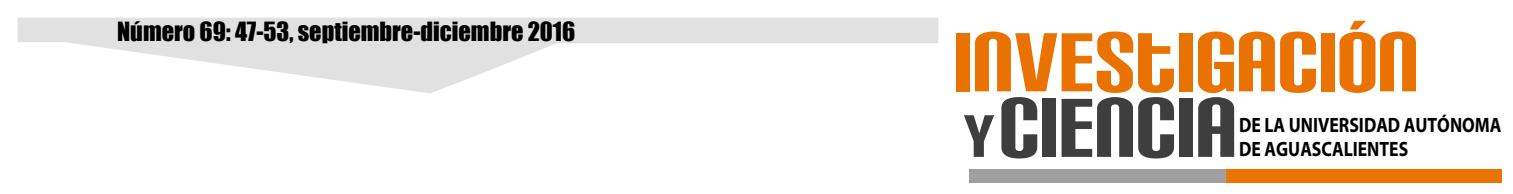

\title{
Estrategia de negocio, gestión de recursos humanos y desempeño organizacional
}



RESUMEN

Dentro del enfoque estratégico organizacional, en los últimos años se ha enfatizado el papel de los recursos humanos en el establecimiento de la estrategia competitiva, el crecimiento organizacional y la creación de valor. Por ello, el presente estudio busca explorar el vínculo entre la estrategia de negocio, las prácticas de gestión de recursos humanos (GRH) y el desempeño organizacional, en una muestra de PyME del sector de manufactura del estado de Querétaro. Los resultados muestran que existen diferencias en las prácticas de GRH en función de la estrategia de negocio adoptada, los valores más altos se encontraron en estrategias prospectoras y analizadoras. Igualmente, se halla que estas estrategias y prácticas están relacionadas positivamente $e$ inciden de manera significativa en el desempeño organizacional. Se concluye que implementar las mismas es importante para crear y mantener el capital humano que permita obtener un mejor desempeño y alcanzar una ventaja competitiva.

\section{ABSTRACT}

In recent years, the organizational strategic approach has emphasized the role of human resources in the

Palabras clave: estrategia, gestión, recurso humano, desempeño, prácticas, PyME.

Keywords: strategy, management, human resources, performance, practices, SMEs.

Recibido: 8 de septiembre de 2015, aceptado: 7 de junio de 2016

División Industrial, Universidad Tecnológica de Querétaro.

Facultad de Ingeniería, Universidad Autónoma de San Luis Potosí.

Autor para correspondencia: bynzunza@uteq.edu.mx establishment of competitive strategy, organizational growth and value creation. So that, the present study aims to explore the linkages between business strategy, human resources management practices (HRM) and organizational performance in a sample of 116 manufacturing SMEs in the state of Queretaro. The results show that there are differences in human resource management practices (HRM) in terms of the business strategy adopted, reaching the highest values in prospectors and analyzers strategies. It was also found that these strategies and practices are positively related and have a significant impact on organizational performance. It is concluded that implementing them is important to build and maintain the human capital that allows to get a better performance and achieve a competitive advantage.

\section{INTRODUCCIÓN}

En la literatura reciente, los recursos humanos han sido identificados por ser particularmente importantes para lograr un desempeño mejorado y exitoso, considerando que apoyan e incrementan las capacidades organizacionales que sustentan y dan forma a la estrategia de negocio y permiten a las organizaciones responder rápidamente a las amenazas $u$ oportunidades ambientales. También se ha mencionado que en combinación con otros activos permiten obtener una ventaja competitiva, ya que proveen el soporte para hacer estrategia, agregan valor a los procesos productivos y facilitan el desarrollo de capacidades gerenciales (Huselid et al., 1997; Andersen y Minbaeva, 2013).

De la misma forma, se han señalado las diferencias e interacción existentes entre los tipos de 
estrategia, las prácticas de GRH (Schuler y Jackson, 1987) y la necesidad de que las organizaciones cuenten con sistemas de gestión de recursos humanos (SGRH) que fomenten el desarrollo de capital humano; las habilidades, capacidades y motivación para trabajar y alcanzar las metas organizacionales (Hamid, 2013). Sin embargo, y aunque el estudio de estas prácticas y su relación con el desempeño ha llamado mucho la atención, poco se sabe del papel que la estrategia de negocio puede jugar en esta relación, particularmente en empresas pequeñas.

Así, el objetivo de este estudio es explorar el impacto de la estrategia de negocio y las prácticas de GRH sobre el desempeño organizacional y contribuir mediante evidencia empírica al conocimiento de esta relación considerando una muestra de PyME del sector de manufactura; así como proveer información clave para la optimización de los procesos estratégicos de estas organizaciones. Con base en ello se formula la pregunta de investigación siguiente: ¿ Cuál es el vínculo existente entre la estrategia de negocio, las prácticas de GRH y el desempeño organizacional? También se detalla la estructura del trabajo, que inicia con los antecedentes del tema, revisión de literatura y metodología de investigación, resultados y discusión de sus implicaciones teóricas y prácticas, así como conclusiones, limitaciones y propuestas de investigación futuras.

\section{Revisión de literatura}

Diversas investigaciones han planteado que la adopción de ciertas prácticas de GRH puede mejorar el desempeño y la competitividad en las empresas pequeñas (Michie y Sheehan, 2008; Razouk, 2011). Asimismo, se ha sugerido que la implementación de una estrategia organizacional exitosa demanda el desarrollo de políticas y prácticas de GRH que alienten la creación y retención del capital humano, además de ser una fuente sostenible de ventaja competitiva (Huselid et al., 1997; Barney y Wright, 1998). De igual forma se ha referido la relación entre la estrategia de negocio, el desempeño organizacional y estas prácticas (Miles y Snow, 1984; Porter 1985; Michie y Sheehan, 2008; Lee et al., 2010), y se ha encontrado que las empresas que siguen determinadas estrategias, de acuerdo a diferentes tipologías, logran un desempeño superior (Miles et al., 1978; Karami et al., 2015; Ho, 2016). Otro dato encontrado es que gracias a la estrategia las políticas y prácticas pueden vincularse con los objetivos de negocio y el contexto externo de las organizaciones, lo que contribuye en diferentes formas al desempeño de las mismas (Wangithi Waiganjo et al., 2012). Estas referencias teóricas sustentan la siguiente hipótesis:

H1: Existe un vínculo significativo y positivo entre la estrategia de negocio y el desempeño organizacional.

La revisión de literatura evidencia también el vínculo y las diferencias existentes en las prácticas de GRH en función de la estrategia implementada. Por ejemplo, organizaciones con un marcado énfasis hacia el desarrollo de recursos internos, la selección y el reclutamiento interno, sistemas basados en salarios fijos y una nula evaluación del desempeño; otras que se inclinan a contratar gente sin experiencia, a proveer capacitación y priorizan el desarrollo de habilidades y resultados (Miles y Snow, 1984; Peck, 1994), por lo que optan por sistemas más desarrollados de administración de recursos humanos; organizaciones que prestan poca atención a algunas prácticas como las de entrenamiento y desarrollo, mediante una estrategia de reducción de costos; o las que persiguen una estrategia de innovación que priorizan estas mismas (Katou y Budhwar, 2010), las cuales tienden a otorgar mayores incentivos para contar con personal con las mayores competencias.

Sin embargo, existe poco consenso sobre cuáles prácticas de GRH pueden ser consideradas estratégicas. Algunos refieren el entrenamiento y desarrollo, el reclutamiento y selección, la evaluación y compensación del desempeño, el desarrollo de carrera, la comunicación interna, los sistemas de recompensa y trabajo de alto desempeño, el empleo selectivo y el trabajo en equipo, entre otros (Lee et al., 2010; Rasouk, 2011; Hamid, 2013; Loo See y Leap Han, 2013; Sheehan, 2014). Estas aportaciones permiten sustentar la hipótesis siguiente:

H2: Existe un vínculo significativo y positivo entre las estrategias de negocio y las prácticas de GRH.

Se ha encontrado que las prácticas de GRH se correlacionan significativamente con el desempeño organizacional representado por indicadores de productividad, rentabilidad, ventas, satisfacción de clientes y lealtad de los empleados (Wright et al., 2005; Wattanasupachoke, 2009; Úbeda García et al., 2013; Karami et al., 2015; Altarawneh, 2016; Peregrino De Brito y Barbosa De Oliveira, 2016). También se ha probado el impacto de algunas de ellas sobre el 
desarrollo de recursos estratégicos asociados con el desempeño y la ventaja competitiva (Huselid et al., 1997; Wright et al., 2005; Wattanasupachoke, 2009; Hamid, 2013; Peregrino De Brito y Barbosa De Oliveira, 2016). Además, plantean que su efecto es mayor sobre el desempeño cuando las mismas soportan la estrategia de negocio, por lo que se propone la hipótesis presentada a continuación:

H3: Existe un vínculo significativo y positivo entre las prácticas de GRH y el desempeño organizacional.

\section{MATERIALES Y MÉTODOS}

El estudio fue de tipo transversal causal. La población objeto de estudio está conformada por pequeñas y medianas empresas de la zona metropolitana del estado de Querétaro. La investigación tiene como unidad de análisis al sector de manufactura industrial. La muestra alcanzada fue de 116 empresas, lo que representa $17.96 \%$ de la población total. El muestreo fue no probabilístico por conveniencia. La recopilación de información se realizó mediante entrevista personalizada a directivos, a través de un cuestionario de 50 ítems. La misma se llevó a cabo durante el primer semestre de 2014. La escala utilizada fue tipo Likert de 5 puntos. Las características de las organizaciones que participaron en la investigación se sintetizan en la Tabla 1.

En cuanto a los constructos que forman parte del estudio se emplearon las escalas desarrolladas por Miles y Snow (1984) que evalúan la estrategia con base en cuatro arquetipos: prospectores, analizadores, defensores y reactores; de Peck (1994) y Wright et al. (2005), que miden las prácticas de reclutamiento y selección, entrenamiento y capacitación, desarrollo interno, así como la evaluación y compensación del desempeño; Venkatraman (1989) y Vorhies y Morgan (2005), que consideran medidas de desempeño. Estas escalas fueron ajustadas y validadas en el contexto local, eliminando solo 2 ítems de la estrategia y 1 del desempeño. Esto en virtud de la estructura y carga factorial. Para clasificar las organizaciones en función de la estrategia se realizó un análisis clúster jerárquico con el método Ward. La Tabla 2 resume los resultados de los grupos obtenidos. En ella se observa que las empresas con estrategias analizadoras y prospectoras obtienen valores superiores a la media global en todas las variables exploradas en comparación con estrategias defensoras y reactoras. En virtud de estos hallazgos, solo se seleccionaron estas estrategias para realizar el análisis estructural considerando que parecieran ser las que representan mejor los constructos estudiados y se ha probado que organizaciones con este tipo de estrategias cuentan con SGRH más desarrollados (Miles y Snow, 1984; Peck, 1994).

\section{RESULTADOS}

Para contrastar las hipótesis planteadas se hizo uso del análisis estructural (SEM) con el programa AMOS. La consistencia interna de la escala de medición con el Alfa de Cronbach fue de 0.947. La varianza total explicada fue de $70.22 \%$. La fiabilidad y validez de las dimensiones de los constructos se realizó mediante el análisis factorial confirmatorio (AFC). Los valores obtenidos de consistencia interna, validez

Tabla 1. Características de la unidad muestral

\begin{tabular}{|c|c|c|c|c|c|c|}
\hline \multirow[t]{2}{*}{ Sector } & $\begin{array}{c}\text { Química, caucho y } \\
\text { plástico }\end{array}$ & $\begin{array}{c}\text { Metal } \\
\text { mecánica }\end{array}$ & $\begin{array}{c}\text { Alimentos, bebidas y } \\
\text { tabacos }\end{array}$ & Textil & Servicios & $\begin{array}{c}\text { Otros } \\
\text { minoritarios }\end{array}$ \\
\hline & $23.3 \%$ & $40.5 \%$ & $10.3 \%$ & $6.9 \%$ & $8.6 \%$ & $10.3 \%$ \\
\hline Tamaño & \multicolumn{2}{|c|}{$23.3 \%$} & \multicolumn{4}{|c|}{$76.7 \%$} \\
\hline $\begin{array}{l}\text { Tipo de } \\
\text { empresa }\end{array}$ & \multicolumn{2}{|c|}{ Familiar } & \multicolumn{2}{|c|}{ Sociedad independiente } & \multicolumn{2}{|c|}{ Subsidiaria } \\
\hline \multirow{2}{*}{$\begin{array}{l}\text { Origen del } \\
\text { capital }\end{array}$} & \multicolumn{2}{|c|}{ Nacional } & \multicolumn{2}{|c|}{ Extranjero } & \multicolumn{2}{|c|}{ Mixto } \\
\hline & \multicolumn{2}{|c|}{$41.4 \%$} & \multicolumn{2}{|l|}{$53.4 \%$} & \multicolumn{2}{|c|}{$5.2 \%$} \\
\hline \multirow{2}{*}{ Mercado } & \multicolumn{2}{|c|}{ Nacional } & \multicolumn{2}{|c|}{ Extranjero } & \multicolumn{2}{|c|}{ Mixto } \\
\hline & \multicolumn{2}{|c|}{$32.8 \%$} & \multicolumn{2}{|l|}{$12.9 \%$} & \multicolumn{2}{|c|}{$54.3 \%$} \\
\hline
\end{tabular}

Elaboración propia. 
Tabla 2. Clasificación de las organizaciones por estrategia de negocio

\begin{tabular}{|c|c|c|c|c|c|c|}
\hline \multirow{2}{*}{\multicolumn{2}{|c|}{\begin{tabular}{|l|} 
Estrategia de negocio \\
\end{tabular}}} & Grupo 1 & Grupo 3 & Grupo 3 & Grupo 4 & \\
\hline & & Defensora & Reactora & Analizadora & Prospectora & Total \\
\hline \multicolumn{2}{|c|}{ No. de empresas } & 26 & 8 & 28 & 54 & 116 \\
\hline \multicolumn{2}{|l|}{ Porcentaje } & $23 \%$ & $7 \%$ & $23 \%$ & $47 \%$ & $100 \%$ \\
\hline \multirow{5}{*}{$\begin{array}{l}\text { Prácticas de } \\
\text { GRH }\end{array}$} & Reclutamiento y selección & 3.63 & 1.87 & 4.26 & 4.37 & 3.53 \\
\hline & $\begin{array}{l}\text { Entrenamiento y } \\
\text { capacitación }\end{array}$ & 2.59 & 1.88 & 4.04 & 4.43 & 3.23 \\
\hline & Desarrollo interno & 1.93 & 1.78 & 2.71 & 4.13 & 2.63 \\
\hline & $\begin{array}{l}\text { Evaluación del } \\
\text { desempeño }\end{array}$ & 2.44 & 1.63 & 4.00 & 4.56 & 3.16 \\
\hline & $\begin{array}{l}\text { Compensación del } \\
\text { desempeño }\end{array}$ & 2.85 & 1.50 & 3.70 & 4.39 & 3.11 \\
\hline \multirow{4}{*}{ Desempeño } & Crecimiento & 3.04 & 3.50 & 3.78 & 4.13 & 3.61 \\
\hline & Rentabilidad & 2.81 & 3.40 & 3.85 & 3.85 & 3.50 \\
\hline & Operación & 3.52 & 4.13 & 4.30 & 4.24 & 4.05 \\
\hline & Satisfacción del cliente & 3.31 & 3.69 & 4.28 & 4.21 & 3.87 \\
\hline \multirow[t]{2}{*}{ Total } & Suma GRH & 13.44 & 8.66 & 18.71 & 21.88 & 15.67 \\
\hline & Suma DO & 12.68 & 14.72 & 16.21 & 16.43 & 15.01 \\
\hline
\end{tabular}

Elaboración propia.

Tabla 3. Consistencia interna y validez convergente del modelo teórico

\begin{tabular}{|c|c|c|c|c|c|c|c|}
\hline Variables & Indicadores & $\begin{array}{l}\text { Carga } \\
\text { factorial }\end{array}$ & $\underset{\text { R2 }}{\text { Fiabi. }}$ & $\begin{array}{l}\text { Valor } t \\
\text { robusto }\end{array}$ & $\begin{array}{l}\text { Alfa de } \\
\text { Cronb. }\end{array}$ & $\begin{array}{c}\text { Índice } \\
\text { Fiab. } \\
\text { Comp. }\end{array}$ & $\begin{array}{c}\text { Índice } \\
\text { Var. } \\
\text { Extr. }\end{array}$ \\
\hline $\begin{array}{l}\text { Estrategia de } \\
\text { negocio }\end{array}$ & $\begin{array}{l}\text { ESTPRO } \\
\text { ESTANA }\end{array}$ & $\begin{array}{l}.728^{* * *} \\
.914^{* * *}\end{array}$ & $\begin{array}{l}.530 \\
.835\end{array}$ & $\begin{array}{l}1.000 \\
6.565\end{array}$ & .694 & 0.809 & 0.682 \\
\hline $\begin{array}{l}\text { Gestión de } \\
\text { recursos humanos }\end{array}$ & $\begin{array}{l}\text { COMPDPÑO } \\
\text { RECYSELEC } \\
\text { ENTYCAP } \\
\text { DESAINT } \\
\text { EVALDPÑO }\end{array}$ & $\begin{array}{l}.790^{* * *} \\
.678^{* * *} \\
.881^{* * *} \\
.667^{* * *} \\
.783^{* * *}\end{array}$ & $\begin{array}{l}.625 \\
.459 \\
.777 \\
.445 \\
.614 \\
\end{array}$ & $\begin{array}{l}1.000 \\
7.577 \\
9.971 \\
7.401 \\
8.769\end{array}$ & .940 & 0.822 & 0.539 \\
\hline $\begin{array}{l}\text { Desempeño } \\
\text { organizacional }\end{array}$ & $\begin{array}{l}\text { DOREN } \\
\text { DOOPE } \\
\text { DOSAT } \\
\text { DOCRE }\end{array}$ & $\begin{array}{l}.713^{* * *} \\
.643^{* * *} \\
.876^{* * *} \\
.686^{* * *}\end{array}$ & $\begin{array}{l}.509 \\
.414 \\
.767 \\
.471\end{array}$ & $\begin{array}{l}1.000 \\
6.327 \\
7.498 \\
9.386\end{array}$ & .924 & 0.8948 & 0.5835 \\
\hline
\end{tabular}

Nota: Valores de referencia: cargas factoriales $>0.6$ y significativas $(p<0.001)$; IFC $>.7$; IVE $>0.5$; valores de $t>$ valor crítico de $t=1.96$ con $p$ $<0.05, t=2.58 \operatorname{con} p<0.01$, o $t=3.29$ con $p<0.001$. Elaboración propia.

convergente y discriminante, al igual que de ajuste del modelo, reflejan que los resultados son aceptables. Ver Tablas 3 y 4.

Los resultados alcanzados mediante la aplicación del análisis estructural (SEM) al igual que los indicadores de ajuste del modelo se muestran en la Tabla 5. En cuanto a la primer hipótesis planteada, la cual busca conocer el vínculo existente entre la estrategia de negocio y el desempeño organizacional,
Tabla 4. Validez discriminante del modelo teórico

\begin{tabular}{|c|c|c|c|}
\hline \multicolumn{4}{|c|}{ Correlaciones entre constructos } \\
\hline Variable & EN & GRH & DO \\
\hline EN & .682 & .342 & .383 \\
\hline GRH & $.541-.629$ & .539 & .370 \\
\hline DO & $.573-.699$ & $.503-.715$ & .583 \\
\hline
\end{tabular}


Tabla 5. Resultados del modelo estructural y prueba de hipótesis

\begin{tabular}{|cc|c|c|c|}
\hline \multicolumn{2}{|c|}{ Relación estructural } & $\begin{array}{c}\text { Coeficiente } \\
\text { estandarizado }\end{array}$ & Valor $\boldsymbol{t}$ robusto & Prueba de hipótesis \\
\hline H1: EN & DO & $.399^{* *}$ & 3.347 & Aceptada \\
\hline H2: EN & GRH & $.585^{* * *}$ & 5.121 & Aceptada \\
\hline H3: GRH & DO & $.376^{* *}$ & 3.310 & Aceptada \\
\hline
\end{tabular}

S-BX2 $(\mathrm{df}=40)=55.245 ; \mathrm{p}<0.055 ; \mathrm{NFI}=.920 ; \mathrm{CFI}=.976 ; \mathrm{IFI}=.977 ; \mathrm{RMR}=0.49 ; \mathrm{RMSEA}=.058 .{ }^{* * *}=<0.001$, $* *=<0.01$. Elaboración propia.

la asociación es significativa con un efecto considerado como medio $(\beta=.399 ; p<0.01)$, lo que prueba la relación existente entre estos constructos. El segundo supuesto explora la relación entre las prácticas de GRH y el desempeño organizacional, la cual es significativa con un efecto medio $(\beta=.376$; $p<0.01$ ). Los estimados de las dimensiones fueron todas positivas y significativas con efectos grandes, lo que valida la importancia de que las organizaciones desarrollen estos recursos y capacidades. La tercera hipótesis evalúa el vínculo entre la estrategia de negocio y las prácticas de GRH. El mismo es positivo y significativo ( $\beta .585, p<0.001$ ). Es decir, la estrategia de negocio afecta de manera importante las prácticas de GRH, lo cual puede repercutir positiva o negativamente en el desempeño organizacional.

\section{DISCUSION}

El propósito de esta investigación fue explorar la relación de la estrategia de negocio y las prácticas de GRH con el desempeño organizacional. El estudio probó que el reclutamiento y selección, el entrenamiento y la capacitación, la evaluación y compensación del desempeño, así como el desarrollo interno, tienen un efecto significativo sobre el desempeño organizacional. Los hallazgos encontrados son importantes porque proporcionan evidencia del papel estratégico de las prácticas de GRH para el éxito organizacional, lo que sugeriría que una mayor inversión en sistemas y prácticas de GRH es una estrategia apropiada para que se obtengan no solo mejores resultados, sino también una ventaja competitiva.

En segundo lugar, el estudio provee evidencia empírica del efecto mediador de la estrategia de negocio sobre las prácticas de GRH y el desempeño organizacional, lo que contribuye a la investigación previa sobre la relevancia de estas estrategias y prácticas. Igualmente de las diferencias existentes en función del tipo de estrategia de negocio implementada; esto al demostrar empíricamente que las empresas con estrategias prospectoras y analizadoras tasan mejor en todas las prácticas de GRH evaluadas, sobre todo en las relacionadas con la evaluación y compensación del desempeño, así como el entrenamiento y la capacitación. Otros autores han encontrado también diferencias entre algunas de estas con distintas estrategias (Miles y Snow, 1984; Schuler y Jackson, 1987; Peck, 1994).

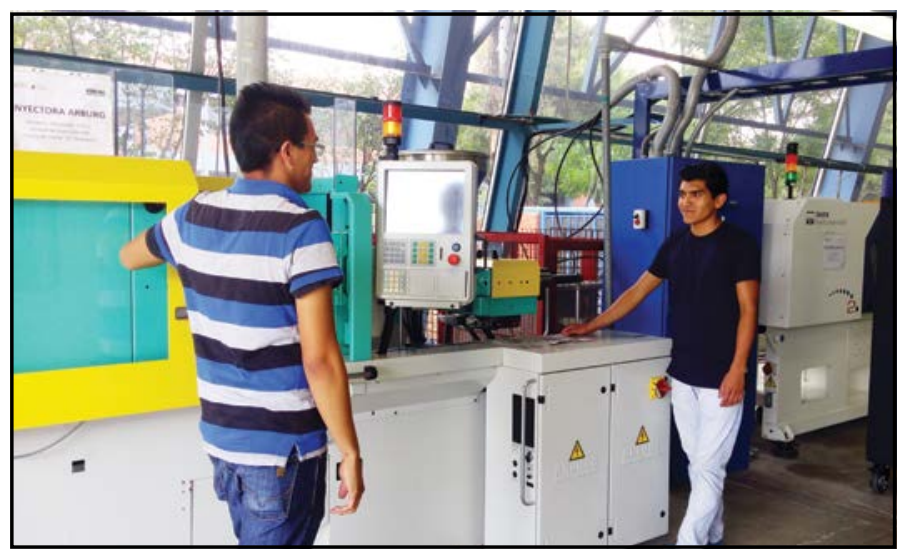

Figura 1. La creación de valor en las organizaciones a través del recurso humano. Fotografía tomada por los autores.

En cuanto a los supuestos planteados, se prueba la relación que existe entre la estrategia de negocio y las prácticas de GRH. Estos hallazgos son consistentes con estudios previos, en los cuales se han abordado las mismas prácticas (Miles et al., 1978; Katou y Budhwar, 2010; Lee et al., 2010), donde han sido las mejores predictoras del desempeño a la evaluación y compensación, al igual que al desarrollo interno (Loo See y Leap Han, 2013; Úbeda García et al., 2013; Altarawneh, 2016). De ahí que sean estas las prácticas en las que debieran focalizarse los sistemas de gestión de recursos humanos.

La segunda hipótesis confirma el vínculo positivo y significativo entre la estrategia de negocio y el desempeño organizacional, lo cual coincide con 
los resultados alcanzados por Miles et al. (1978) y Lee et al. (2010), quienes hallan diferencias entre la implementación de estrategias prospectoras y analizadoras vs. defensoras y reactoras. Por ello, lograr la correcta alineación entre las primeras y las prácticas de GRH pareciera ser un aspecto clave, ya que este tipo de estrategias demandan recursos humanos con habilidades y competencias distintas, y satisfacer las mismas dependerá en gran medida del establecimiento de SGRH que propicien el desarrollo de las mismas. Finalmente, el tercer supuesto prueba la relación positiva y significativa entre las prácticas de GRH y el desempeño organizacional, lo que aporta suficiente soporte empírico con respecto al papel estratégico que juegan los recursos humanos en el desempeño organizacional. Otros investigadores han incursionado en el estudio de estos mismos constructos y han alcanzado resultados similares al probar el efecto de estas prácticas en los resultados organizacionales (Huselid et al., 1997; Lee et al., 2010; Sheehan, 2014). De ahí deriva la necesidad de fortalecer los SGRH y evaluar la efectividad de los mismos, considerando que los empleados tienen el potencial de incrementar el desempeño y ser una fuente crítica de ventaja competitiva.

\section{CONCLUSIONES}

Los resultados de esta investigación permiten concluir que el desempeño organizacional de las PyME manufactureras del estado de Querétaro depende en gran medida de una adecuada gestión del recurso humano y del establecimiento de estrategias de negocio que propicien el desarrollo del mismo; de igual forma, de los recursos que se destinen para la inversión en el factor humano como un medio para seleccionar, desarrollar y retener al personal con las mejores habilidades, capacidades y conocimientos, a la par de sistemas y prácticas que fortalezcan sus competencias, niveles de satisfacción y retribución.

Otro aspecto importante es la capacidad con que las PyME manufactureras de Querétaro logren desarrollar e implementar sistemas de gestión de recursos humanos efectivos y compatibles con la estrategia de negocio, ya que estos son factores críticos para el funcionamiento y éxito de una organización. Ahí radica la importancia de su gestión dentro de los procesos estratégicos de la empresa.

Los resultados obtenidos tienen otras implicaciones, como el efecto que la estrategia de negocio tiene en la gestión de los recursos humanos para la construcción de otros recursos y capacidades nucleares vinculados directamente con el desempeño, como la optimización de los procesos, el desarrollo de tecnología y la innovación. Igualmente, la selección de estrategias de negocio prospectoras y analizadoras que parecen favorecer un comportamiento proactivo e innovador; además del desarrollo de SGRH que garanticen implementar exitosamente la estrategia de negocio, lo que contribuye a mejorar la posición competitiva y supervivencia de las organizaciones.

Se recomienda a los directivos de las organizaciones el fortalecimiento de sus sistemas de gestión de recursos humanos, aunado al establecimiento de mecanismos de medición y retroalimentación que validen la pertinencia de los mismos, el establecimiento de estrategias de actuación, así como la implementación de estrategias prospectoras y analizadoras, ya que ambos parecen agregar valor a las organizaciones y ser fuente de ventaja competitiva; a las entidades gubernamentales y educativas relacionadas con las PyME, soportar el desarrollo de programas de mejora relacionados con la gestión del recurso humano.

Dentro de las limitaciones de este estudio están el tamaño de la muestra, lo cual reduce la generalización de los resultados al contexto explorado. En futuras investigaciones podría ampliarse la misma y profundizar en el conocimiento de estas organizaciones con un estudio longitudinal. También podrían explorarse otras prácticas como el trabajo en equipo, la seguridad y flexibilidad laboral, y construirse escalas más robustas. Igualmente, incursionar en el estudio de otras variables que pudiesen afectar el establecimiento y efectividad de los sistemas, como el estilo de liderazgo o la cultura organizacional, el tamaño y edad de la empresa. 
LITERATURA CITADA

- ALTARAWNEH, I. I. Strategic human resources management and its impact on performance: the case from Saudi Arabia. International Journal of Business Management and Economic Research, 7(1): 486-503, 2016.

- ANDERSEN, T. J. y MINBAEVA, D. The role of human resource management in strategy making. Human Resource Management, 52(5): 809-827, 2013.

- BARNEY, J. y WRIGHT, P. On becoming a strategic partner: the role of human resources in gaining competitive advantage. Human Resource Management, 37(1): 31-46, 1998.

- HAMID, J. Strategic human resource management and performance: the universalistic approach-case of Tunisia. Journal of Business Studies Quarterly, 5(2): 184-201, 2013.

- HO, P. Analysis of competitive environments, business strategies, and performance in Hong Kong's construction industry. Journal of Management in Engineering, 32(2): 1-14, 2016.

- HUSELID, M. A. et al. Technical and strategic human resources management effectiveness as determinants of firm performance. Academy of Management Journal, 40(1): 171$188,1997$.

- KARAMI, A. et al. The influence of HR practices on business strategy and firm performance: the case of banking industry in Iran. The IUP Journal of Management Research, 14(1): 30-53, 2015.

- KATOU, A. A. y BUDHWAR, P. S. Causal relationship between HRM policies and organizational performance: evidence from the greek manufacturing sector. European Management Journal, 28(1): 25-39, 2010.

- LEE, F. H. et al. The relationship between human resource management from steel practices, business strategy and firm performance: evidence industry in Taiwan. The international Journal of Human Resource Management, 21 (9): 1351-1372, 2010.

- LOO SEE, B. y LEAP HAN, L. Human resource management best practices and firm performance: a universalistic perspective approach. Serbian Journal of Management, 8(2): 155-167, 2013

- MICHIE, J. y SHEEHAN, M. Human resource management and corporate performance: evidence from UK and US small firms. En R. Barrett \& S. Mayson (Eds.), International Handbook of Entrepreneurship and HRM (137-164). Cheltenham: Edward Elgar, 2008.

- MILES, R. E. y SNOW, C. C. Designing strategic human resource systems. Organizational Dynamics, 31 (1): 36-52, 1984.
- MILES, R. E. et al. Organizational strategy, structure, and process. New York, US: McGraw-Hill, 1978.

- PECK, S. R. Exploring the Link between Organizational Strategy and the Employment Relationship: The Role of Human Resources Policies. Journal of Management Studies, 31 (5): 715 736, 1994

- PEREGRINO DE BRITO, R. y BARBOSA DE OLIVEIRA, L. The Relationship between Human Resource Management and Organizational Performance. Brazilian Business Review, 13(3): 90-110, 2016.

- PORTER, M. E. The Competitive Advantage: Creating and Sustaining Superior Performance. NY, US: Free Press, 1985.

- RAZOUK, A. A. High-performance work systems and performance of french small- and medium-sized enterprises: examining causal order. International Journal of Human Resource Management, 22(2): 31 1-330, 2011.

- SCHULER R. S. y JACKSON, S. E. Linking competitive strategies with human resource management practices. Academy of Management Executive, 1 (3): 207-219, 1987.

- SHEEHAN, M. Human resource management and performance: evidence from small and medium sized firms. International Small Business Journal, 32 (5): 545-570, 2014.

- ÚBEDA GARCÍA, M. et al. Training policy and organizational performance in the spanish hotel industry. The International Journal of Human Resource Management, 24(15): 2851-2875, 2013.

- VENKATRAMAN, N. Strategic orientation of business enterprises: the construct, dimensionality and measurement. Management Science, 35(8): 942-962, 1989.

- VORHIES, W. D. y MORGAN, N. A. Benchmarking marketing capabilities for sustainable competitive advantage. Journal of Marketing, 69(1): 80-94, 2005.

- WANGITHI WAIGANJO, E. et al. Relationship between strategic human resource management and firm performance of Kenya's corporate organizations. International Journal of Humanities and Social Science, 2(10): 62-70, 2012

- WATTANASUPACHOKE, T. Strategic human resource management and organizational performance: a study of thai enterprises. Journal of Global Business Issues, 3(2): 139-148, 2009.

- WRIGHT, P. M. et al. The relationship between HR practices and firm performance: examining causal order. Personnel Psychology, 58(2): 409-446, 2005. 